\title{
Molecularly Imprinted Solid-Phase Extraction for Determination of Enrofloxacin and Ciprofloxacin in Chicken Muscle
}

\author{
Hongyuan Yan and Kyung Ho Row \\ Department of Chemical Engineering. Inha Lnversity, Incheon t02-751, Korea. ${ }^{\circ}$ E-mail: rowhorinha.ack \\ Received February 11, 2008
}

\begin{abstract}
A simple and sensitive high-performance liquid chromatographic method was developed for the simultaneous identification of enrofloxacin and its active metabolite ciprofloxacin in chicken muscle. Norflorxacin imprinted polymers synthesized in water-containing systems show high selectivity to enrofloxacin and ciprofloxacin in an aqueous environment. Using these water-compatible imprinted polymers as selective adsorbents in the solid-phase extraction of enrofloxacin and ciprofloxacin from chicken samples. the remaining biological matrix could be quickly washed out from the imprinted column while enrofloxacin and ciprofloxacin were selectively retained and enriched. Analytical separation was performed on a $\mathrm{C}_{18}$ column using acetonitrile-water as a mobile phase and fluorescence detection. Good linearity was obtained from 0.8 to $500 \mathrm{ng} / \mathrm{g}(r>0.998)$ with relative standard deviation of less than $3.9 \%$. The mean recoveries of enrofloxacin and ciprofloxacin from chicken muscle were $80.6-94.5 \%$ and $77.8-91.8 \%$ at three different concentrations. The limits of determinations based on $\mathrm{S} / \mathrm{N}=3$ were $0.07 \mathrm{ng} / \mathrm{g}$ and $0.09 \mathrm{ng} / \mathrm{g}$. which are below the maximum residue limits established in many countries.
\end{abstract}

Key Words : Molecularly imprinted polymer. Selective solid-phase extraction. Enrofloxacin. Ciprofloxacin. Chicken muscle

\section{Introduction}

Enrofloxacin (ENR) is a fluoroquinolone with a broad antibacterial spectrum and high bactericidal activity against major pathogenic bacteria found in diseased animals. ${ }^{1-3}$ The pharmacokinetics of ENR is characterized by good absorption and extensive distribution into various animal fluids and tissues. In several animal species. including chicken. ENR is de-ethylated to its primary metabolite. ciprofloxacin (CPX). and both ENR and CPX are found in the muscle and tissue of animals receiving ENR. ${ }^{+-6}$ The widespread administration of these drugs in veterinary medicine represents a potential risk. because their residues may persist in edible animal tissues and may result in the development of drug-resistant bacterial strains or allergies. ${ }^{7.8}$ Therefore. the determination of their residues in meat and other animal products (e.g. milk. eggs) used for human consumption is an important task. Several methods for detection and quantification of ENR and CPX in biological fluids and tissues have been proposed. such as an immunosorbent assay ${ }^{9}$ voltammetry: spectrophotometry. ${ }^{1]}$ capillary electrophoresis. ${ }^{12-14}$ and liquid chromatographic methods. ${ }^{15}-18$ Most of these methods involve a preliminary extraction step followed by a second clean-up step with liquid-liquid extraction or solid-phase extraction (SPE). As such. these approaches are complicated. time-consuming. and use large amounts of organic solvents. Moreover. the low selectivity caused by the endogenous components may contribute to interference with drugs.

Molecular imprinting is a rapidly developing technique for the preparation of polymers having specific molecular recognition properties for a given compound its analogues. or for a single enantiomer. ${ }^{19-21}$ Molecularly imprinted polymers
(MIPs) possess several advantages over their biological counterparts including low cost. ease of preparation. storage stability high mechanical strength, and applicability in harsh chenical media. ${ }^{22.23}$ As a technique for the creation of artificial receptor-like binding sites with a 'memory' for the shape and functional group positions of the template molecule. the application of MIPs has drawn extensive attention and MIPs have been used successfully in many fields of chemistry and biology. particularly as selective adsorbents for solid phase extraction. ${ }^{2+-27}$ However in the majority of MIPs applications. optimum MIPs synthesis and molecular recognitions occurs in non-polar or low polar șistemı. The presence of polar solvents. especially water. can disturb the formation of the prepolymerization complex during imprinting procedure. which obvious limits their further application in environmental and biological matrices.

Tuiel et $\mathrm{al}^{2}{ }^{2}$ synthesized MIPs using dichloromethane and methanol as polar porogenic solvent. which exhibit recognition properties under aqueous conditions. However. the use of additional sample pretreatment procedures was required to remove harmful matrix components and suppress the nonspecific binding. Caro et al ${ }^{29.30}$ prepared ENR and CPX MIPs by bulk polymerization using dichloromethane as porogenic solvent and applied it as a selective sorbent in a two-step SPE (a commercial Oasis cartridge and a molecularly imprinted SPE cartridge were combined) to extract fluoroquinolone from biological samples. However. these procedures are complicated and drugs may be partially lost in the sample preparation steps.

In the present work. water-compatible MIPs were developed in water-methanol șystems using norflorxacin as a dummy template and 2-hỵdroxyethỵl methacrỵlate as a monomer for 
selective extraction of ENR and CPX from chicken muscle samples. The obtained imprinted polymers show high affinity to ENR and CPX in aqueous media and were successfully applied as special SPE sorbents for selective extraction and separation of ENR and CPX from chicken muscle samples. This method is a viable alternative tool to the existing HPLC methods for analyzing the residuals of these quinolones in biological sample

\section{Experimental Section}

Materials. Norflorxacin. enrofloxacin. and ciprofloxacin were obtained from Sigma (St Louis. MO. USA) and their molecular structures are shown in Figure 1. 2-hydroxyethyl methacrylate was purchased from Aldrich (Steinheim. Germany) and purified by distillation. Ethylene glycol dimethacrylate (EDMA) was obtained from Tohyo Kasei Kogyo Co., LTD. (Tokyo, Japan) and was extracted with 2.0 $\mathrm{mol} / \mathrm{L}$ sodium hydroxide solution and dried over anhydroxide magnesium sulfate. $\alpha_{n} \alpha^{\prime}$-Azobis (isobutyronitrile) (AIBN) was purchased from Jumsei Chenical Co., Ltd. (Tokyo. Japan) and recrystallized prior to use. Acetonitrile. tetrahydrofuran. and methanol were all of HPLC grade and obtained from Duksan Pure Chenical Co. LTD (Ansan. Korea). All the other reagents used in the experiment were of the highest grade commercially available. Double deionized water was filtered with a $0.45 \mu \mathrm{m}$ filter membrane before use.

HPLC analysis. HPLC analysis was performed using a Shimadzu HPLC system equipped with a LC-10A Multisolvent Delivery System, a DGU-12A on-line-degasser. a SCL-10Avp gradient controller, a CTO-10Avp column thermostat, and a RF-10AXI fluorescence detector (Shimadzu. Kyoto, Japan). Excitation and emission wavelengths were set at $280 \mathrm{~nm}$ and $480 \mathrm{~nm}$. respectively. A CLASS-VP workstation (Shimadzl, Kyoto. Japan) was used as a data acquisition system. The analytical column was packed with ODS $\mathrm{C}_{18}$ stationary phase (VP-ODS. $150 \mathrm{~mm} \times 4.6 \mathrm{~mm}$ I.D. particle size $5 \mu \mathrm{m}$. Shimadzu. Japan). The column thermostat was set at $30^{\circ} \mathrm{C}$. The mobile phase was wateracetonitrile $(92: 8, \mathrm{v} / \mathrm{v}$, containing $0.1 \%$ trifluoroacetic acid) and the flow rate was set at $1.0 \mathrm{~mL} / \mathrm{min}$.

Preparation of molecularly imprinted polymers. Norflorxacin imprinted polymers were prepared by thermalinitiated polymerization within a $25 \mathrm{~mL}$ thick-walled glass tube. The polymerization mixture was composed of 1.0 mmol norflorxacin. $8.0 \mathrm{mmol}$ 2-hy'droxyethỵl methacrylate.
30.0 nmol EDMA, and $0.06 \mathrm{~g}$ AIBN. dissolved in appropriate porogenic solvents (methanol:water $=4: 1 . \mathrm{v} / \mathrm{v}$ ). The solution was sonicated for $10 \mathrm{~min}$ and purged with helium gas for $10 \mathrm{~min}$ before being sealed inside the tube under a helium environment. Polymerization was performed at a temperature of $55^{\circ} \mathrm{C}$ in a water bath for $20 \mathrm{~h}$. After the polymerization. the polymers were grinded and sieved to obtain imprinted particles in a range of $28 \mu \mathrm{m}-32 \mu \mathrm{m}$. and then suspended in acetone until the upper solution became clear. Finally. the particles were dried under vacuum and put into a column and washed with tetrahydrofuran. methanolacetic acid-trifluoracetic acid $(80: 19.5: 0.5 \mathrm{v} / \mathrm{v})$ to remove the templates. After washing with methanol and being dried in a drying oven $\left(45^{\circ} \mathrm{C}\right)$, the particles were stored for further use. Non-imprinted blank polymers (NIP, in the absence of a template) were prepared and treated in an identical manner.

Determination of the binding property of the imprinted Polymers. In order to investigate the binding capacity of the MIPs in water enviromments, a static absorption experiment and a Scatchard analysis were employed. $20 \mathrm{mg}$ of imprinted polymer and $3.0 \mathrm{~mL}$ of aqueous solution were placed into $5.0 \mathrm{~mL}$ flasks and oscillated in darkness at room temperature for $24 \mathrm{~h}$. The concentration of ENR in the aqueous solution was varied from 0 to $3.5 \mathrm{mmol} / \mathrm{L}$. The solutions were centrifuged and filtered and a free concentration analysis was subsequently performed via HPLC. The absorption quantity $(Q)$ was calculated by subtracting the free concentration from the initial concentration.

Morphological characteristics of the MIPs. Morphological characteristics, including results of a pore analysis and SEM analysis of the polymers, were also investigated in this experiment. The specific surface areas and porosity of the imprinted polymers were obtained by measuring nitrogen adsorption on an ASAP 2000 accelerated surface area and porosimetry sy stem (Micromeritics. USA). The NIP was analyzed as a control experiment to demonstrate the differences between imprinted and non-imprinted polymers. A microscopic analysis of the MIPs was carried out using an S-4200 Scanning Electron Microscope (Hitachi, Japan) at $15 \mathrm{kV}$.

Procedure of molecularly imprinted solid phase extraction. $100 \mathrm{mg}$ of the imprinted particles and blank particles was respectively packed in an empty polypropylene cartridge and preconditioned with $5.0 \mathrm{~mL}$ methanol and 5.0 $\mathrm{mL}$ water. The following sample pretreatment was modified on the basis of a previously reported method. ${ }^{18,31}$ Briefly. the chicken muscle samples were vortexed for $2.0 \mathrm{~min}$ with phosphate buffer to produce an extraction solution and then
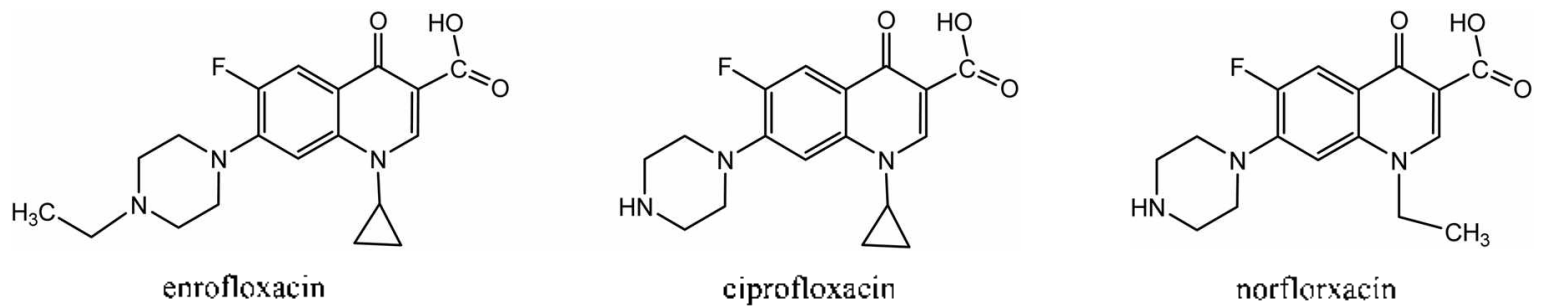

Figure 1. Molecular structures of enrofloxacin, ciprofloxacin, and norflorxacin. 
centrifuged for $5 \mathrm{~min}$ at $10000 \mathrm{rpm}$; these steps were repeated three times. The supernatant was percolated into a MISPE cartridge for cleanup (washing with $5.0 \mathrm{~mL}$ of aqueous solution and elution with $4.0 \mathrm{~mL}$ of acetonitriletrifluoracetic acid $(99: 1 . \mathrm{v} / \mathrm{v})$ ). and the collected eluate was subsequently filtered through a cellulose acetate filter $(0.20$ im pore size, Advantec MFS Inc. CA. USA). The filtrate were evaporated to dryness under a gentle stream of helium at $45^{\circ} \mathrm{C}$ and reconstituted in $0.5 \mathrm{~mL}$ of mobile phase before injection to the HPLC system.

Stock standard solutions of fluoroquinolones were prepared in water. Spiked nuscle samples were prepared by adding appropriate volumes of the fluoroquinolone standard solution to blank muscle samples; the volume added was maintained at less than $2 \%$ of the final sample volume to preserve the integrity of the samples. After vortex mixing for $10 \mathrm{~min}$, the mixture was equilibrated for $60 \mathrm{~min}$ at $37^{\circ} \mathrm{C}$ in darkness and then extracted according to the above MISPE procedure

\section{Results and Discussion}

Preparation and characteristic of the MIPs. In the majority of MIP-based applications. optimum molecular recognition is attained in aprotic and low polar organic solvents. often in the solvent used in the polymerization process. The presence of polar solvents. especially water, can disturb the formation of the prepolymerization complex during the inprinting procedure, and interactions between monomers and the template are also easily disrupted. In order to synthesize MIPs that demonstrate specific recognition for ENR and CPX in a water environment. MIPs synthesized in a water-containing system were investigated using different proportions of methanol-water as a porogenic solvent and their recognition ability in an aqueous system was evaluated. Schematic illustrations of the imprint formation and molecular recognition processes are shown in Figure 2. It was found that the proportion of water in the polymerization mixtures has a critical effect on the pore properties and the surface area of the resulting polymers. The reason for this is that. as a porogenic solvent. water not only brings all the components (template. functional monomers. crosslinker and initiator) into one phase but also creates macropore structures in the imprinted polymers. Meanwhile. methanol-water $(4: 1, \mathrm{v} / \mathrm{v})$ provided sufficient rigidity and desirable surface properties in the obtained polymers (Fig. 3). Further increase of the water content would result in a flexible polymer with a small surface area. Conversely. lower water content resulted in a high density polymer with small pore size. Furthermore. the MIPs using 2-hydroxyethyl methacrylate as a monomer showed higher recognition of the target molecule than the MIPs prepared using acrylamide and methacrylic acid due to the fomer's stronger electrostatic and ion-exchange interactions with the target in a water environment. In addition. because the ENR suffers degradation processes under UV irradiation. thermal-initiated polymerization was applied in this work.

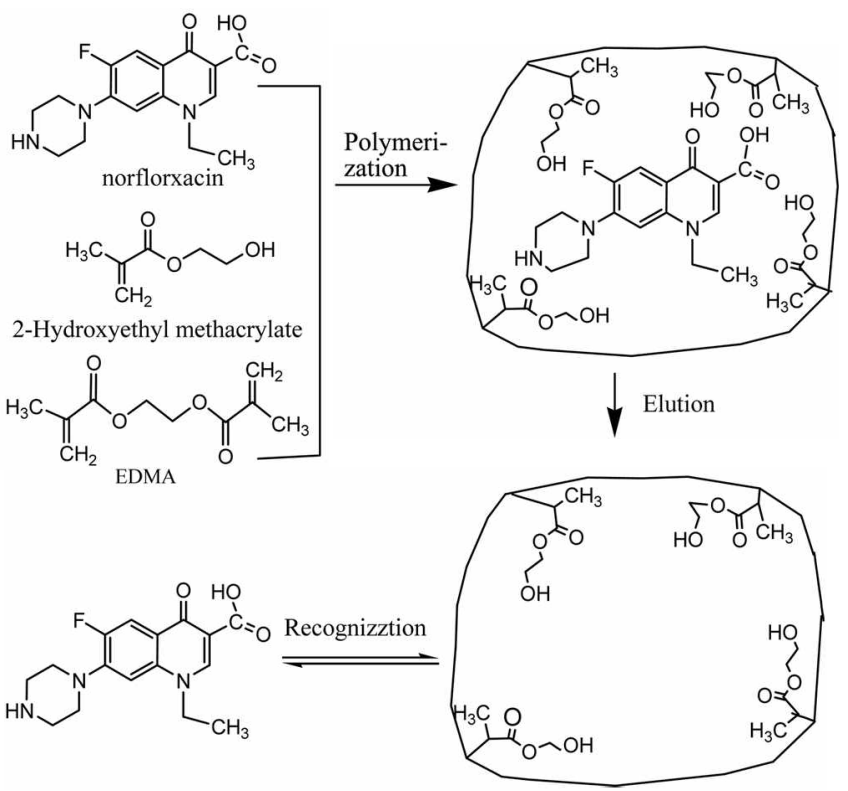

Figure 2. Schematic illustrations of the imprint fonmation and molecular recognition processes.

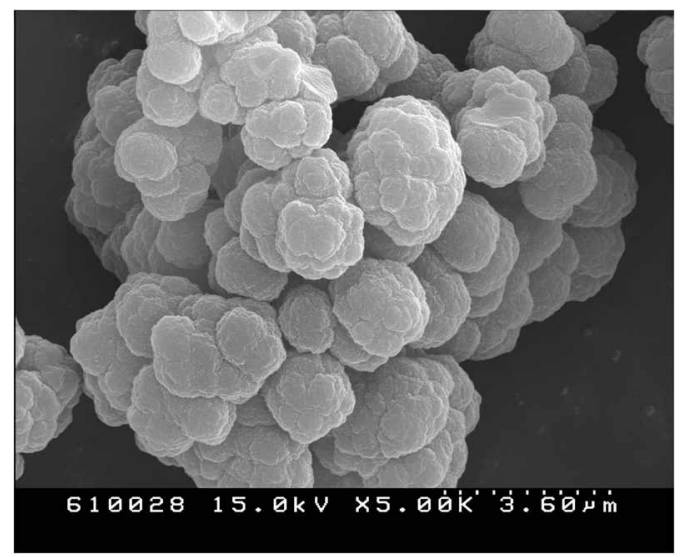

Figure 3. Scanning electron microscope (SEM) inage of the imprinted particles

Binding properties of the MIPs. The maximum binding capacity and dissociation constant were employed to evaluate the binding properties of the MIPs. Data obtained from a static absorption experiment were further processed with the Scatchard equation:

$$
Q / C_{\text {free }}=\left(Q_{\text {max }}-Q\right) / K_{D}
$$

where $Q$ is the amount of ENR bound to MIPs at equilibrium. $Q_{\max }$ is the maximum binding capacity, $C_{\text {fie }}$ is the free analyte concentration at equilibrium, and $K_{D}$ is the dissociation constant. As shown in Figure 4 , the Scatchard plot was not a single straight line, suggesting that the binding sites in the MIPs are heterogeneous with respect to their affinity for ENR. There are two distinct sections within the plot that can be regarded as straight lines, and hence the binding sites can be classified into two distinet groups with specific binding properties. The respective $K_{D}$ and $Q_{\text {max }}$ values are calculated from the slopes and intercepts of the two linear portions of the results of the Scatchard analysis and are listed in lable 1 . The specific surface areas and 


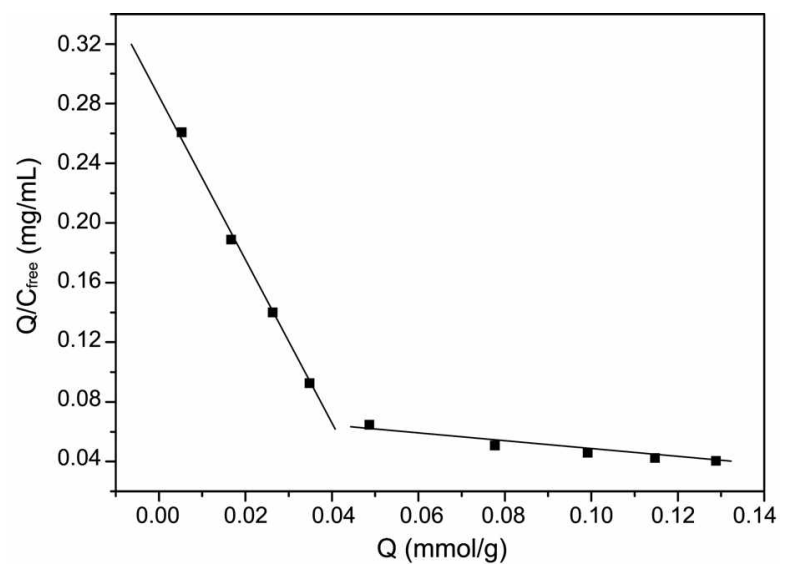

Figure 4. Scatchard andalysis of the MIPs.

Table 1. The results of Scatchard Analysis

\begin{tabular}{cccc}
\hline Binding sites & Linearity & $K_{D}$ & $\begin{array}{c}Q_{\max } \\
(\mathrm{mmol} / \mathrm{g})\end{array}$ \\
\hline Higher affunity site & $O / C_{\mathrm{ftee}}=0.288-5.637 \mathrm{Q}$ & 0.177 & 0.051 \\
Lower afturity site & $O / C_{\text {fiet }}=0.0767-0.297 \mathrm{Q}$ & 3.364 & 0.258 \\
\hline
\end{tabular}

pore volumes from the nitrogen adsorption experiments were $291 \mathrm{~m}^{2} / \mathrm{g}$ and $0.562 \mathrm{~cm}^{3} / \mathrm{g}$ for MIP and $279 \mathrm{~m}^{2} / \mathrm{g}$ and $0.559 \mathrm{~cm}^{3} / \mathrm{g}$ for NIP, respectively. The similar surface areas and pore volumes of MIP and NIP indicate that the selectivity of the MIP could be ascribed to special imprinted recognition.

Chromatographic evaluation of the MIPs. The obtained MIPs particles were put into a HPLC column (200 mm- 4.6 $\mathrm{mm}$ I.D.) for further chromatographic evaluation. ENR and CPX cannot be washed out from the column within $60 \mathrm{~min}$ when acetonitrile. methanol. or water is used as a mobile phase. However. ENR and CPX could be washed out from the blank column in less than 15 min. which indicates that the retention ability of the MIPs for the fluoroquinolones could be attributed to special imprinted recognition. Furthermore. with a decrease of the $\mathrm{pH}$ of the mobile phase, the special affinity was destroyed and ENR and CPX could be washed out. These results can be understood in that both hydrophobic and ion-exchange interactions contribute to the retention of the analytes on the imprinted polymers. The strongly $\mathrm{pH}$-dependence due to ENR and CPX are hydrophilic zwitterions. comprising 6-fluoro. 7-piperazinyl. and 3carboxyl groups. Because of the acid-basic characteristics of ENR and CPX, they can be present in an aqueous solution in cationic, anionic. or intermediate forms due to the presence of a carboxylic group and the charged anino group of the piperazine moiety. When the portion of trifluoracetic acid in the mobile phase is above $0.5 \%$. ENR and CPX could be eluted within 5 minutes. These values, taken together with the elution profiles. demonstrate that the MIPs have high affinity for ENR and CPX and the retention ability can be easily controlled by adjusting the $\mathrm{pH}$ of the mobile phase. These characteristics lend the MIPs potential applicability as selective adsorbents for use in the enrichment, separation. and/or detection of ENR and CPX in biological and environmental samples.
Sample preparation of chicken muscle. One of the main difficulties in the development of an analytical method for a complex biological matrix is the sample pretreatment, which becomes more complicated when several analytes with different chemical properties are implied. The existing methods typically involve SPE after protein precipitation using organic solvents in conjunction with strong organic or inorganic acids. These conditions can have different effects on the retention of fluoroquinolones in the selected solidphase cartridge. In this study, the sample pretreatment was modified on the basis of a previously reported method, and satisfactory recoveries ( $77.8 \%-94.5 \%$ ) were obtained using a phosphate buffer as an extraction solution followed by MISPE. During optimization of the sample preparation procedure. different vortex and centrifuging times were evaluated in an effort to obtain the best recovery and least preparation time. It was found that vortexing for $2.0 \mathrm{~min}$ and centrifugation of $5 \mathrm{~min}$ yield satisfactory recoveries, and further increase of the vortex and centrifugation times does not further increase the recoveries. Compared with reported extraction procedures (including protein precipitation, defatting, and other complex treatments). the sample preparation procedure required less organic solvents and fewer
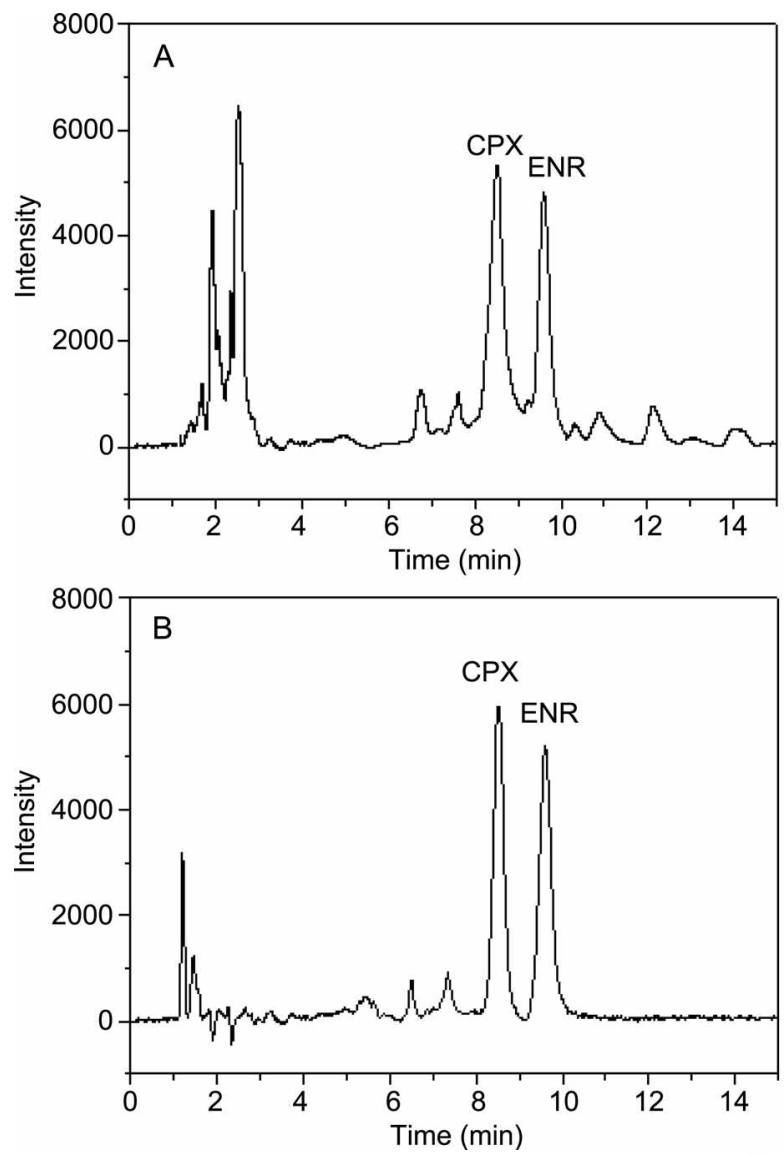

Figure 5. Chromatogram of spiked chicken sample by difterent extract methods. (A: SPE with $\mathrm{C}_{18}$ adsorbent; B: MISPE (imprinted adsorbent); analytical column: $C_{18} 5 \mu \mathrm{m}, 150 \mathrm{~mm} \times 4.6 \mathrm{~mm}$ I.D.: mobile phase: water-acetonitrile ( $92: 8, v / \%$, containing $0.1 \%$ trifluoroacetic acid); flow rate: $1.0 \mathrm{~mL} / \mathrm{min}$; spiked concentration: $5.0 \mathrm{ng} / \mathrm{g}$; injection volume: $10 \mu \mathrm{L}$ ) 
Table 2. The Recoveries of ENR and CPX in Three Spiked Chicken Samples $(n=5)$

\begin{tabular}{|c|c|c|c|c|c|c|}
\hline \multirow{2}{*}{ Spiked level } & \multicolumn{2}{|c|}{$2.011 \mathrm{~g} / \mathrm{g}$} & \multicolumn{2}{|c|}{$10 \mathrm{ng} / \mathrm{g}$} & \multicolumn{2}{|c|}{$50 \mathrm{ng} / \mathrm{s}$} \\
\hline & Recovery (\%) & $\mathrm{RSD}(\%)$ & Recovery $(\%)$ & $\operatorname{RSD}(\%)$ & Recovery (\%) & $\operatorname{RSD}(\%)$ \\
\hline Enrofloxacin & 80.6 & 58 & 86.4 & 5.3 & 94.5 & 3.1 \\
\hline Ciprofloxacin & 77.8 & 7.6 & 83.7 & 6.1 & 91.8 & 5.4 \\
\hline
\end{tabular}

steps, but good recoveries were still obtained.

The selectivity of molecular imprinting-solid phase extraction (MISPE). Due to their special selectivity for conducting target analyte isolation. MIPs were proved to be a better selection. giving higher recovery values and a clearer

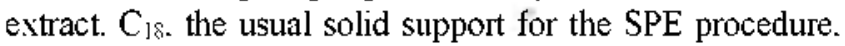
was also investigated and the results showed that the recoveries of ENR and CPX were between $64 \%$ and $82 \%$ with high relative standard deviations. These deviations are attributed to nonspecial interactions such as hydrophobic and hydrophilic interactions between the various components of the sample matrix with $\mathrm{C}_{18}$ sorbents.

The chrontatograms of spiked chicken samples after different SPE processes are shown in Figure 5. The chromatogrants indicate that there were no interferences from the biological matrix after the MISPE process, which demonstrates the high selectivity and affinity of the synthesized MIPs in a water environment. Moreover. the major hurdles of the MISPE application-"template leakage" was avoided by using a molecule with a structure very closely related to the target analyte as the template. which can be distinguished from the target analyte upon subsequent quantification by HPLC. Blank chicken samples were also treated under the same conditions and no peaks were observed in the chromatogram at the same retention times with ENR and $\mathrm{CPX}$. indicating that there was no interference from endogenetic components of the biological matrix. Furthermore. comparison of the relative extraction recoveries (calculated relative to the initial concentration of ENR) for MIP and NIP reveals a significant difference. The relative recovery of ENR on the NIP is similar for each step of the extraction. irrespective of the solvent applied. In contrast. MIPs show high affinity towards the target. and it was necessary to have TFA present in the eluting step to effectively desorb ENR and CPX from the imprinted materials.

Optimization of rinsing and eluting conditions. To optimize the process of selective MISPE. the parameters of washing and eluting step were investigated. It is important to apply a wash step immediately after the extraction in dealing with the biological samples. as this ensures reduction of the matrix interference in the separation of analytes and prevents the protein from polluting the cartridge and analytical column. Because the solvent system should resemble the biological system and based on findings of the earlier chromatographic analysis. water was selected as the rinsing solvent. For the purpose of determining the minimum volume of water able that can efficiently rinse the endogenetic components. different volumes of water were investigated from 1.0 to 8.0 $\mathrm{mL}: 5.0 \mathrm{~mL}$ was found to be the optimum volume of rinsing solvent.

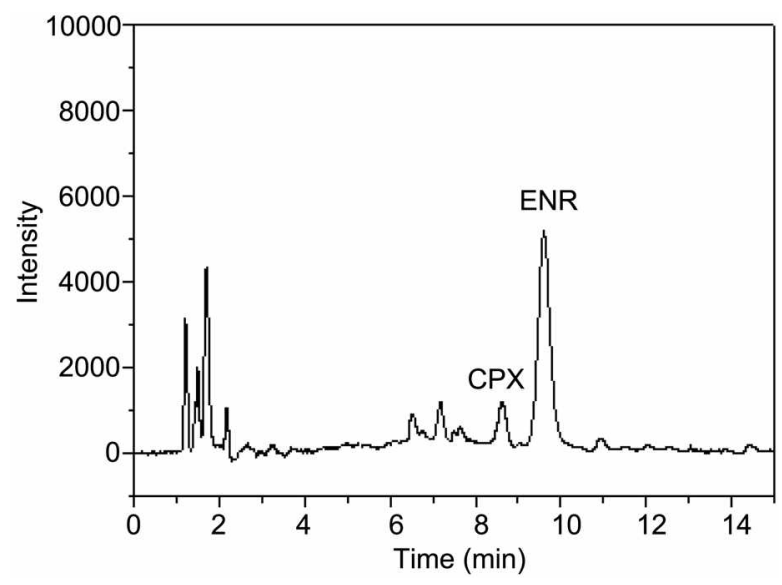

Figure 6. Chromatogram of chicken samples after oral administration. (Oral dose: $15 \mathrm{mg} / \mathrm{kg}$ : sample pretreatment: MLSPE: analytical column: $C_{18}, 5 \mu \mathrm{m}, 150 \mathrm{~mm} \times 4.6 \mathrm{~mm}$ I.D.; mobile phase: water-acetonitrile $(92: 8, v / v$, containing $U .1 \%$ trifluoroacetic acid): flow rate: $1.0 \mathrm{~mL} / \mathrm{min}$; injection volume: $10 \mu \mathrm{L}$ )

In order to obtain the highest recovery for ENR from the chicken samples. a series of elution solutions, water. methanol. and acetonitrile with TFA in different proportions were used to optimize the eluting conditions. The best recovery was obtained by using acetonitrile-trifluoracetic acid (99:1, $\mathrm{v} / \mathrm{v})$ as an eluting solution. With further increase of the eluting strength of acetonitrile-trifluoracetic acid. the recoveries of ENR were nearly constant and additional interference from the biological matrix was observed. After optimization. $4.0 \mathrm{~mL}$ was found to be the optimum volume of elution solution

Validation of the proposed method. Calibration curves were constructed using the areas of the chromatographic peaks measured at nine increasing ENR and CPX concentrations. in a range of 0.8 to $500 \mathrm{ng} / \mathrm{g}$. Good linearity was obtained for all analytes throughout the concentration range. and the following linear correlation equations were obtained: $\mathrm{Y}=1.86 \times 10^{7} \mathrm{X}+1.89 \times 10^{5}$ for ENR and $\mathrm{Y}=1.52 \times 10^{6} \mathrm{X}$ $+1.79 \times 10^{5}$ for CPX with correlation coefficients of $r>$ 0.998 . The precision (inter- and intra-day) and accuracy of the method were assessed using chicken muscle samples spiked with $5 \mathrm{ng} / \mathrm{g}$ and $20 \mathrm{ng} / \mathrm{g}$ concentration levels for each analyte. The intra-day repeatability evaluated as RSD ranged from 3.1 to $7.6 \%$ and the inter-day reproducibility was below $9.5 \%$ in all cases. The mean recoveries of ENR and CPX in chicken samples were evaluated by spiking three different levels $(2.0 .10 .50 \mathrm{ng} / \mathrm{g})$ of standard analyte to samples in replicates of five. The chromatographic peak areas of the analyte were compared to those of standards at the same concentration to provide the recovery values. and 
the results are shown in Table 2 . The linits of deterninations (LOD) were 0.07 and $0.09 \mathrm{ng} / \mathrm{g}$ for ENR and CPX. respectively. based on a signal-to-noise ratio of 3 , and are well below the tolerance levels set by the European Union.

Application to real chicken samples. The applicability of the method was tested with muscle from chickens receiving ENR in a single oral dose at a level of $15 \mathrm{mg} / \mathrm{kg}$ body mass. Using this MISPE-HPLC method, the residual levels of ENR and CPX in chicken muscle could be determined even after three days of administration. A chromatogram of the chicken samples after oral administration is shown in Figure 6.

Twenty chicken muscle samples were randomly collected from local markets and analyzed by the developed MISPEHPLC method. No residue of ENR or CPX was observed in any of the samples, thus demonstrating that misapplication of these kinds of veterinary medicine is not extensive.

\section{Conclusions}

A simple and sensitive MISPE-HPLC assay procedure is described for the sumultaneous determination of ENR and CPX in chicken samples. Norfloxacin intprinted polymers prepared in water-methanol systems show high affinity to ENR and CPX in an aqueous environment and were successfilly applied as selective adsorbents of SPE to extract and separate ENR and CPX from chicken samples. Good linearity was obtained, from 0.8 to $500 \mathrm{ng} / \mathrm{g}(\mu>$ 0.998 ). with relative standard deviations less than $3.9 \%$. The sample preparation procedure in this method requires less organic solvents and fewer steps. but good recoveries. accuracy, and LOD were nevertheless obtained.

Acknowledgements. The authors gratefully acknowledge financial support by the Center for Advanced Bioseparation Teclunology of Inha University. Korea.

\section{References}

1. Mitchell. M. A. J. Exotic Pet Nedicine 2006. 15, 66.

2. Efthiniadou. E. K.: Katsaros. N.: Karaliota, A: Psomas, G. Bioorg. Hed. Chem. Lett. 2007, 17, 1238.

3. Lizondo. M.: Pons. M.: Gallardo. M.: Estelrich. J. J. Pharm. Bioned. Anol. 1997, 15.1845.

4. Rao. G. S.: Ramesh. S.: Almmad. A. H.: Tripathi. H. C.: Sharma. L.
D.: Malik. I. K. Vet. J. 2002. 163.85.

5. Fang. W. H.: Zhou. S.: Yu. H. J.: Hu. L.: Zhou. K.: Liang. S. Aquacuintre 2007. 272. 180.

6. Dimitrova, D. J.: Lashev. L. D.; Yanev. S. G.: Pandova. B. Res. Iet. Sci. 2007. 82. 392.

7. Doerge, D. R.; Bajic. S. Rapid Commun. Mass Spectom. 1995, 9. 1012 .

8. Gorla. N.: Garcia Ovando. H.: Larripa. I. Toxicol. Lett 1999. 104. 43.

9. Huet. A. C.: Chariler. C.: Tittlemier. S. A.; Singh, G.: Benrejeb, S:; Delahaut, P. J. Agric. Food Chem 2006, 5t, 2822.

10. Navalón, A; Blane. R; Reyes, L.: Navas. N.; Vílchez. J. L. Anal. Chint Acto 2002. 454.83.

11. Cester. C. C.: Toutain. P. L. J. Pham Sci. 1997. 86. 1148.

12. Hernández. M.: Aguilar. C.: Borrull. F.: Calull. M. J. Chronatogr: $B$ 2002, 772. 163 .

13. Lara. F. J.: Garcia-Campana. A. M.: Ales-Barrero. F.: BosqueSendra, J. M.; Garcia-Ayuso. L. E. Anal. Chem. 2006, 78, 7665.

14. Barrón. D.: Timénez-Lozano. E.: Cano. J: Barbosa. J. J. Chrontatogr: B 2001. 759.73.

15. Gonźalez. C.: Moreno. L.: Small. ..: Jones. D. G.: Bruni. S. F. Anal. Chim. Acta 2006, 560, 227.

16. Manceau, J.: Gicquel. M.: Laurentie, M.: Sanders. P. J. Chomatog: $B 1999,726.175$.

17. Idown. O. R.: Peggins. J. O. J. Pham Biomed Anal. 2004. 35. 143.

18. Zhao. S.: Jiang. H.: Li. X.: Mi. T.: Li. C.: Shen. J. J. Agric. Food Chem. 2007, 55,3829 .

19. Kempe. M.: Mosbach. K. J. Chronatogr A 1995. 694, 3.

20. Yan. H.: Jin. L: Row, K. H. J. Liq. Chromatogr Rel. Techol. 2005. 28.3147.

21. Cormack. P. A. G. Elorza. A. Z. J. Chronatogr: B 2004. 80t. 173.

22. Vlatakis. G.: Andersson. L. I.: Miller. R.: Mosbach. K. Natue 1993. 361,645 .

23. Lavignac, N.; Allender. C. J; Brain, K. R. Anal. Chim Acta 2004. 510. 139

24. Pichon. V. J. Chrontatogr A 2007.1152.41.

25. Masquee. N.: Marcee. R. M.: Borrull. F. Trends dnal. Chent. 2001. 20.477.

26. Qiao. F.; Sun. H.: Yan, H.: Row: K. H. Chronatographia 2006. 64.625.

27. Caro. E.: Marce. R. M.: Borrull, F.: Cormack, P. A. G.; Sherrington, D. C. Trends Anal. Chem. 2006. 25. 143.

28. Turiel. E.: Martin-Esteban. A.: Tadeo. J. L. J. Chomatogr A 2007. 1172.97

29. Caro. E; Marce, R. M; Cormack. P. A. G.: Sherrington. D. C: Borrull, F. Anat Chim Acta 2006. 562. 145.

30. Caro. E; Marce, R. M; Cormack. P. A. G.: Sherrington. D. C:; Borrull. F. J. Sep. Sci. 2006. 29. 1230.

31. Ramos. M.: Aranda. A.: Garcia. E.: Reuvers. T.: Hooghuis. H. J. Chrontatogr: B 2003. 789.373. 